\title{
Operations Research Models for Computational Biology, Bioinformatics and Medicine
}

\author{
Paola Bertolazzi · Jacek Blazewicz • Metin Turkay
}

Published online: 30 June 2010

(C) Springer Science+Business Media B.V. 2010

This special issue collects five papers that were presented at the Mini EURO Conference on Computational Biology, Bioinformatics and Medicine organized by the EURO Working Group on Operational Research in Computational Biology, Bioinformatics and Medicine (EURO-CBBM). The Conference was held in Rome on September 2008 and included presentations of 61 talks selected on the basis of extended abstracts and three invited talks.

The objective of the conference was to bring together researchers developing and using state-of-the art modeling and optimization approaches to solve problems in computational biology, bioinformatics and medicine. The conference program was planned with the objective of establishing an effective forum for the exchange and for the discussion of current research, issues and future trends in the above mentioned areas.

The meeting was structured into 15 sessions on Protein Structure Analysis, Protein Structure Prediction, Structural Bioinformatics, Motif Recognition, Image Analysis, Dynamic Models, Networks, Data Mining Methods, DNA Sequencing, Optimization and Feature Extraction Methods and Microarray Analysis.

Out of 61 presentations, 11 were evaluated for publication in the special issue. After two rounds of reviewing process, five papers were selected for publication.

The first paper is "A Logic-Based Approach to Polymer Sequence Analysis" by R. Bruni. This paper presents an improved propositional logic based approach for the estimation of protein sequences from mass spectroscopy results. The correspondence

\footnotetext{
P. Bertolazzi

Istituto di Analisi dei Sistemi ed Informatica “Antonio Ruberti”, Rome, Italy

e-mail: bertola@iasi.cnr.it

J. Blazewicz

Poznan University of Technology, Poznan, Poland

M. Turkay $(\bowtie)$

Koc University, Istanbul, Turkey

e-mail: mturkay@ku.edu.tr
} 
between sequences and natural numbers is considered with propositional logic models, so that all sequences up to a certain molecular weight can be implicitly considered in databases, and explicitly computed only when needed. Results on realworld problems show the effectiveness of this approach.

The second paper "Identification of regulatory network motifs from gene expression data" by L. Farina, A. Germani, G. Mavelli and P. Palumbo. addresses the identification of regulatory network motifs by using recent biotechnologies that are able to provide precise measures of gene expression time courses in response to a large variety of internal and environmental perturbations. A mathematical model of the network comprised of system of ODE equations were considered to simulate the behavior of two ubiquitous motifs: the single input motif and the multi output feedforward loop motif. The algorithm has been tested on simulated noisy data assuming the presence of a step-wise regulatory signal. The results indicate that the method is potentially able to robustly discriminate between alternative motifs, thus providing a useful in silico identification tool for the experimenter.

The third paper " Mathematical Programming based Procedure for Breast Cancer Classification" by Y. Masmoudi, H. Chabchoub, S. Hanafi and A. Rebai. presents a hybrid method for breast cancer classification. The classification problem is posed as the minimization of the distance between the components of each cluster and the centers of the clusters. The determination of the cluster centers is addressed by solving the k-means algorithm. Once the centers of each class are determined, the rule of center neighborhood is applied to assign an element to a class. The hybrid approach was validated on benchmark datasets and applied to an original biological dataset on 84 breast cancer tumors. Each tumor was measured for five parameters corresponding to the expression levels of five biomarkers.

The fourth paper "Statistical analysis of retinal tomographic pseudo images for diagnostic purpose" was co-authored by C. Bruni, J. de Juan, C. Ferrone, D. Giannini, N.M. Grzywacz, D. Huang, G. Koch, V. Russo and O. Tan The early detection of retinal anomalies is of great interest for a diagnosis and treatment of related pathologies. Optical Coherence Tomography (OCT) is a non-invasive technique that produces sections of a three dimensional reflectance map, which are pseudo-images of the retinal structure. These pseudo-images are useful in identifying local modifications of reflectance, which visually appear as blots. Stretchedexponential distribution, a two-parameter probability density function, was assumed to describe the pseudo-images from OCT. Spatial-reflectance interdependence was analyzed by studying the autocorrelation function showing a significant correlation between contiguous pixels about $5 \mu \mathrm{m}$ apart. A procedure is based on the maximumlikelihood estimate of distribution parameters along the retinal pseudo-image to analyze their spatial dependence both along and across retinal layers. It was shown that along suitable thin layers, the two parameters were statistically constant. A statistical procedure was developed for automatic detection of parameter variations due to retinal pathological blots. Some results are reported for a detection of diabetic retinopathy.

The last paper "Combining Gene Expression Pro_les and Drug Activity Patterns Analysis: A Relational Clustering Approach" by E. Fersini, E. Messina, F. Archetti and C. Manfredotti addresses the combined analysis of tissue micro array and drug response to extract valuable knowledge about various relations among gene expressions and drug activity patterns in tumor cells. An approach was developed 
to select a set of most responsive drugs for a given gene expression profile related to a specific tumor tissue. An integrated framework based on a constraint-based clustering algorithm, called Relational K-Means algorithm was used. The algorithm groups cell lines using drug response information and taking into account cell-to-cell relationships derived from their gene expression profiles.

We are grateful to the authors and reviewers for all their efforts in generating the contributions for this special issue. We also hope that this collection of papers provides the readers with an overview of operations research models and algorithms in computational biology, bioinformatics and medicine. 